\title{
An ongoing measles outbreak in the Federation of Bosnia and Herzegovina, 2014 to 2015
}

\author{
M Hukic (mirsadahukic@yahoo.com) ${ }^{1,2}$, J Ravlija3, S Karakas4, M Mulaomerovic ${ }^{3}$, A Dedeic Ljubovic ${ }^{5}$, I Salimović-Besic ${ }^{5}$, M \\ Seremet $^{5}$, S Ahmetagic ${ }^{6}$, A Comor ${ }^{7}$, E Feric ${ }^{2}$ \\ 1. Department of Medical Science, Academy of Sciences and Arts of Bosnia and Herzegovina, Sarajevo, Bosnia and Herzegovina \\ 2. International Burch University, Sarajevo, Bosnia and Herzegovina \\ 3. Institute of Public Health of Federation Bosnia and Herzegovina, Sarajevo, Bosnia and Herzegovina \\ 4. Institute of Public Health of Central Bosnia Canton, Travnik, Bosnia and Herzegovina \\ 5. Department of Clinical Microbiology, University Clinical Centre Sarajevo, Bosnia and Herzegovina \\ 6. Clinic for Infectious Diseases, University Clinical Centre Tuzla, Tuzla, Bosnia and Herzegovina \\ 7. Institute for Biomedical Diagnostic and Research "Nalaz", Sarajevo, Bosnia and Herzegovina
}

Citation style for this article:

Hukic M, Ravlija J, Karakas S, Mulaomerovic M, Dedeic Ljubovic A, Salimović-Besic I, Seremet M, Ahmetagic S, Comor A, Feric E. An ongoing measles outbreak in the Federation of Bosnia and Herzegovina, 2014 to 2015. Euro Surveill. 2015;20(9):pii=21047. Available online: http://www.eurosurveillance.org/ViewArticle. aspx?Articleld $=21047$

Article submitted on 05 February 2015/ published on 05 March 2015

Between January 2014 and the beginning of February 2015, the Federal Institute of Public Health in the Federation of Bosnia and Herzegovina has reported 3,804 measles cases. Notable transmission has been observed in three Central Bosnia Canton municipalities: Bugojno, Fojnica and Travnik. Most cases were unvaccinated $2,680(70 \%)$ or of unknown vaccination status 755 (20\%). Health authorities have been checking vaccination records and performing necessary prevention measures. The epidemic is still ongoing.

Since the beginning of 2014 , a measles outbreak is taking place in the Federation of Bosnia and Herzegovina (FB\&H), including a total of 3,804 measles cases up to the start of February 2015.

The first two cases were reported in Bugojno, Central Bosnia Canton, in siblings who were teenagers, both of whom had respectively visited the local health care centre in early February 2014, with a rash that had started three days earlier. They had recently travelled to Germany.

\section{Description of the outbreak}

For the investigation of the outbreak, the general principles of the case definition of the European Union (EU) Commission Decision of 2012 were used [1]. Laboratory investigations of initial patients were conducted at the Department of Microbiology, University Clinical CentreSarajevo, Bosnia and Herzegovina, and were based on serological findings of the measles virus specific antibody response in serum samples.

From the first municipality affected by the outbreak, Bugojno in Central Bosnia Canton, where school-aged children and adolescents (6 to 19 years-old) with measles were reported from February 2014, the outbreak subsequently spread, in two distinct epidemic waves, to other cantons, including, consecutively, Sarajevo, Zenica-Doboj, Tuzla, Una-Sana, and HerzegovinaNeretva (Figure 1).

\section{FIGURE 1}

Cumulative number and geographical distribution of notified cases of the measles outbreak, Federation of Bosnia and Herzegovina, January 2014-February 2015 $(\mathrm{n}=3,804$ cases $)$

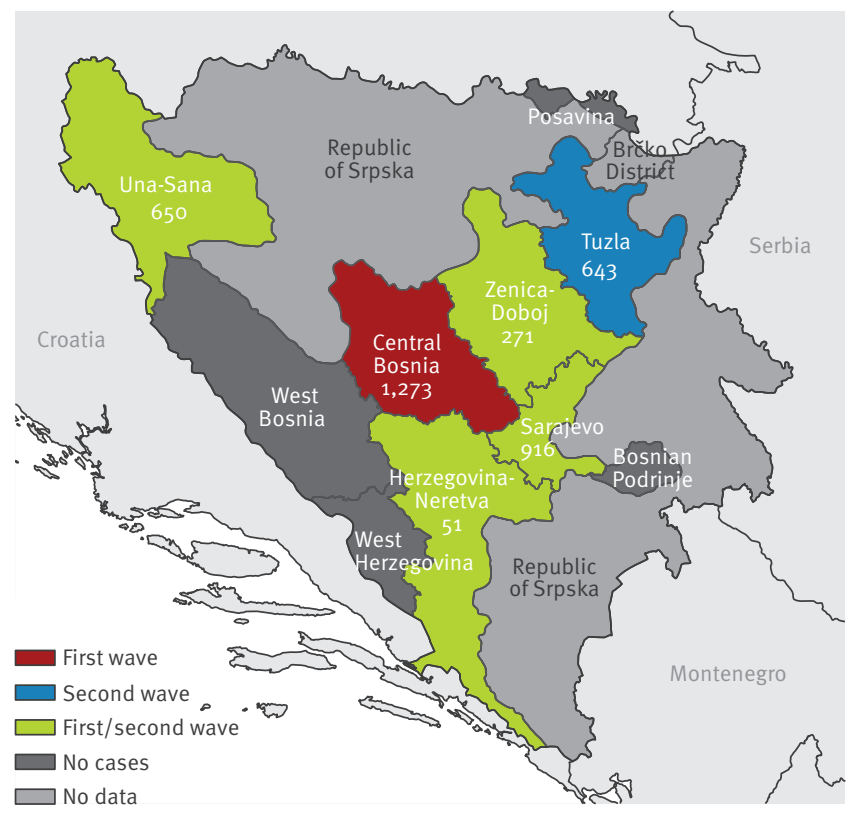

The name of the cantons of the Federation of Bosnia and Herzegovina are in white font, with the number of cases below.

In the legend, the first and second waves, refer to the two waves of the measles outbreak. 
Reported measles cases by week of rash onset, Federation of Bosnia and Herzegovina, January 2014-February 2015 $(\mathrm{n}=3,804)$

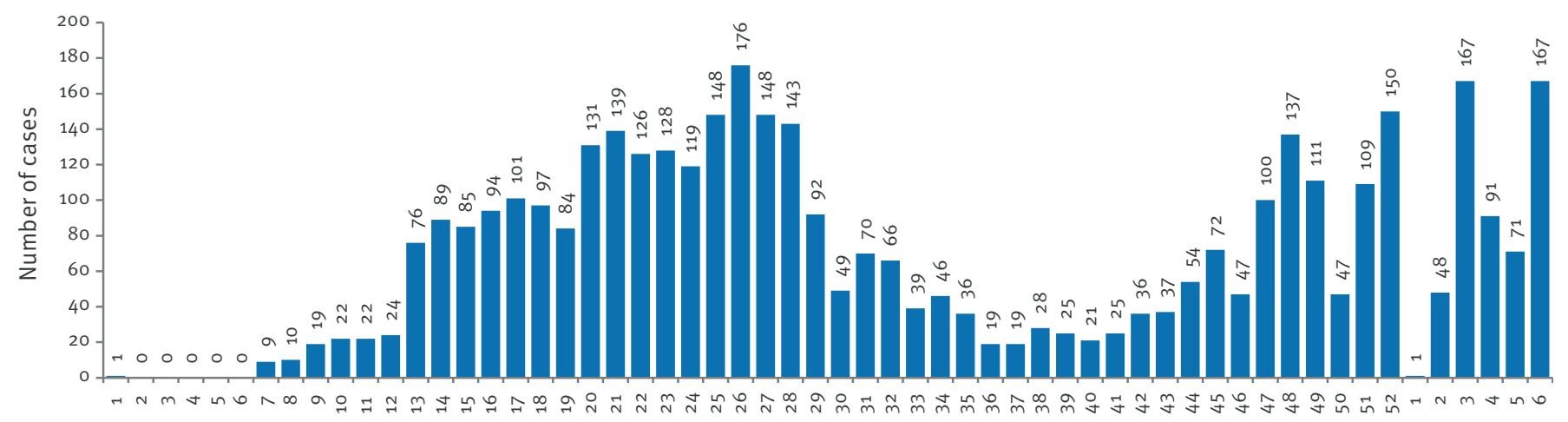

Week number 2014-2015

The first epidemic wave of the outbreak had a peak in week 26 of 2014 (23-29 June, $n=175$ cases) and the second, a peak in week 4 of 2015 (29-25 January, $n=167$ cases) (Figure2). During the first wave which occurred from the beginning of 2014 until July of that year, 2,201 measles cases were reported, mainly in the cantons of Central Bosnia, Zenica-Doboj and Sarajevo. The second wave, from August 2014 to February 2015, accounted for an additional 1,603 cases, mainly in the Tuzla and Una-Sana Cantons. During the second wave, cases continued to occur in the three cantons that were previously most affected (Figure 2).

\section{Age and sex distribution of cases}

Overall, most cases 3,300 ( $87 \%$ ) were under the age of 30 years. The highest number of cases $(n=713)$ were in children aged between 15 and 19 years, followed by one to four year-olds ( $n=637$ cases) and five to nine year-olds ( $n=578$ cases) (Figure 3 ).

With the exception of those aged 30 years and older, for which of a total of 503 affected, 266 were female, more male individuals were reported in each age group (Figure 3).

\section{Vaccination status of cases}

The majority of the outbreak cases had not been vaccinated against measles. Only $2 \%(58 / 3,804)$ had received a full course of vaccination (two doses of the measles, mumps, and rubella (MMR) vaccine), $8 \%(311 / 3,804)$ received one dose, while $70 \%$ $(2,680 / 3,804)$ were unvaccinated. For 755 (20\%) cases vaccination status was unknown.

\section{Laboratory findings}

Nasopharyngeal swabs were sent to the European Regional Reference Laboratory for Measles and Rubella in Luxembourg for genotyping. Investigations of three initial cases revealed the presence of the D8 measles virus genotype, and more samples are currently being analysed.

\section{Control measures}

Catch-up vaccinations have been conducted for schoolaged children and adolescents who had not received two doses of MMR vaccine (the minimum interval between the two doses was four weeks). In addition urgent immunisation campaigns were planned/partially conducted in municipalities where members of the Roma community were affected, as for parts of this community vaccination coverage is low in FB\&H (data not shown). To prevent further spread, and to control the epidemic, persons with measles were asked to stay at home and vaccinations of unvaccinated contacts were carried out in families, kindergartens, schools, etc. according to the national regulations. In total, 1,577 first doses and 3,110 second doses of MMR vaccine were administered, however an obstacle to reaching sufficient vaccination coverage stems from parents following the anti-vaccination movement.

\section{Discussion}

Part of the World Health Organization strategic plan for the control of measles has been its elimination in Europe by 2015 [2]. However, the number of notified measles outbreaks especially in central and western Europe has been increasing in the last five years, with a reported peak in 2011 (32,124 cases) [3-5]. Several countries reported a considerable number of cases, including: France, Bulgaria, Germany, Italy, Romania, Spain, Ukraine, and the United Kingdom [4-6]. According to a report from the European Centre for Disease Prevention and Control, 30 EU/European Economic Area (EEA) countries conducting measles surveillance reported a total of 3,840 cases between December 2013 and November 2014 [6]. The ongoing outbreak of measles in FB\&H accounts for 3,804 cases, highlighting the region as a European hot spot for the disease.

In order to achieve $95 \%$ immunity in the population for measles, vaccination coverage with two doses needs to be higher than $95 \%$. However, this was not achieved in the EU [5], and, similarly, FB\&H has accumulated 
a large unvaccinated population over a long period of time. Vaccination coverage in FB\&H is measured as the percentage vaccinated of the target population (12 months and 6 years). MMR vaccine coverage between 1998 and 2015 in FB\&H ranged from 80.7\% (1999) to $96.2 \%$ (2007) (average value: $87.1 \pm 4.12$ ) for primary immunisation and from $53 \%$ (2006) to $91.9 \%$ (2008) (average value: $82.9 \pm 8.83$ ) for the second dose. Disruption in the immunisation programme during the war (1992-1995) and in the post-war period (19961998) left a considerable number of children susceptible to measles, as well as mumps and rubella $[7,8]$.

The probable causes of the outbreak described here, as well as its expansion, are insufficient vaccination and implementation of proposed control measures. The majority of those affected had not received necessary vaccination (two doses of MMR) at the recommended time (up to 14 years of age). Our data demonstrate that most cases in the current outbreak either did not know their vaccination status (20\%) or reported being either partially ( $8 \%)$ or not vaccinated at all $(70 \%)$.

In 2007, the measles genotype circulating in FB\&H was D4 [9], however in the current outbreak genotype D8 was found, a genotype reported in the western part of Europe (England, Germany, Italy) at the end of 2011 [10]. It cannot be ruled out that the genotype D8 found in this outbreak might have been imported by individuals who travelled to such countries a short time before the beginning of the epidemic $[10,11]$. Molecular epidemiology is an important surveillance tool for routine monitoring of movement and the spreading of different virus genotypes across Europe.

In conclusion, FB\&H is currently facing a large measles outbreak with 3,804 cases by the beginning of 2015 . This is probably related to disruption of routine MMR vaccination during the war and post-war periods, as well as the recent wave of vaccination controversies and the anti-vaccination movement that contribute to parental hesitance and in turn to lower immunisation coverage. Monitoring of the immunisation status and vaccine effectiveness is crucial. High vaccination coverage rates with two doses and advocacy and communication campaigns ensuring effective community involvement and public awareness are necessary to control the current epidemic and to avoid future outbreaks.

\section{Acknowledgements}

The authors are grateful to the Department of Immunology, World Health Organization European Regional Reference Laboratory for Measles and Rubella, Luxembourg Institute of Health/Laboratoire National de Santé, Luxembourg for supporting measles diagnostic in Bosnia and Herzegovina.
FIGURE 3

Age and sex distribution of reported measles cases in the Federation of Bosnia and Herzegovina, January 2014-

February $2015(\mathrm{n}=3,804)$

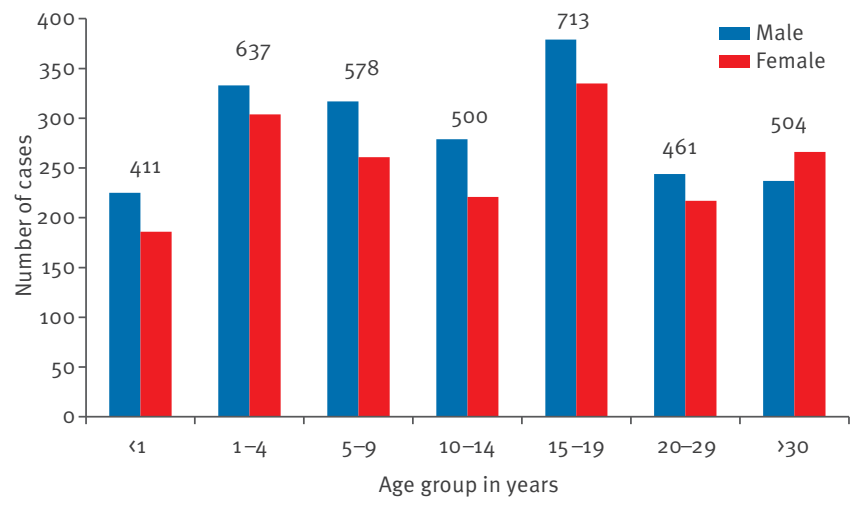

For each age group the total number of cases is indicated above the chart bars.

Conflict of interest

None declared.

Authors' contributions

Mirsada Hukic was involved in the design of the study, drafted the article, analysed and interpreted surveillance data and revised the manuscript. Jelena Ravlija was involved in the analysis and interpretation of epidemiological data. Sead Karakas contributed to the recruitment of study participants and the analysis of surveillance data. Mirsada Mulaomerovic was involved in the analysis of epidemiological data. Amela Dedeic Ljubovic was involved in laboratory investigation and data analysis. Irma Salimovic-Besic contributed to the recruitment of study participants and analysis of surveillance data. Sead Ahmetagic contributed to the recruitment of study participants and analysis of surveillance data. Mensura Seremet was involved in laboratory investigation and data analysis. Aeta Comor was involved in the data interpretation and the revision of the manuscript. Elma Feric was involved in the interpretation of the results and the writing of the manuscript.

References

1. European Centre for Disease Prevention and Control (ECDC). EUVACnet. Case Definition. Measles. Stockholm: ECDC. [Accessed 15 Feb 2015]. Available from: http://ecdc.europa. eu/en/activities/surveillance/euvac/case_definition/Pages/ measles.aspx

2. World Health Organization (WHO). Surveillance Guidelines for Measles, Rubella and Congenital Rubella Syndrome in the WHO European Region. Copenhagen: WHO Regional Office for Europe; 2012.

3. European Centre for Disease Prevention and Control (ECDC). Surveillance Report: Measles and Rubella Monitoring, 2012 Stockholm: ECDC: 2012. Available from: http://www.ecdc. europa.eu/en/publications/Publications/20120709-measles rubellaissue13.pdf

4. World Health Organization Regional Office for Europe (WHO/ Europe). 9th Meeting of the Measles/Rubella Reference Laboratories of the WHO European Region, 10-11 March 2014, Copenhagen, Denmark. Copenhagen: WHO/Europe; 2014 
5. Santibanez S, Prosenc K, Lohr D, Pfaff G, Jordan Markocic 0 , Mankertz A. Measles virus spread initiated at

international mass gatherings in Europe, 2011. Euro Surveill. 2014;19(35):20891. http://dx.doi.org/10.2807/1560-7917.

ES2014.19.35.20891 PMID:25210982

6. European Centre for Disease Prevention and Control (ECDC). Number of measles cases by month and notification rate (cases per million) by country, December 2013 - November 2014,

EU/EEA countries. Stockholm: ECDC. [Accessed 15 Feb 2015]. Available from: http://www.ecdc.europa.eu/en/healthtopics/ measles/epidemiological_data/Pages/measles_past12months. aspx

7. HukicM, HajdarpasicA, RavlijaJ, LerZ, BaljicR, DedeicLjubovicA, et al.Mumps outbreak in the Federation of Bosnia and Herzegovina with large cohorts of susceptibles and genetically diverse strains of genotype G, Bosnia and Herzegovina, December 2010 to September 2012.Euro Surveill. 2014;19(33):20879. http://dx.doi.org/10.2807/1560-7917. ES2014.19.33.20879 PMID:25166347

8. Hukic M,Hübschen JM, Seremet M, Salimovic-Besic I, Mulaomerovic M, Mehinovic N, et al. An outbreak of Rubella in the Federation of Bosnia and Herzegovina between December 2009 and May 2010 indicates failure to vaccinate during war time (1992-1995).Epidemiol Infect. 2012;140(3):447-53. http:// dx.doi.org/10.1017/S0950268811000707 PMID:21524344

9. Kremer JR, Brown KE, Jin L, Santibanez S, Shulga SV, Aboudy Y, et al. High genetic diversity of measles virus, World Health Organization European Region, 2005-2006. Emerg Infect Dis. 2008;14(1):107-14. http://dx.doi.org/10.3201/eid1401.070778 PMID:18258089

10. SantibanezS, HübschenJM, MullerCP, FreymuthF, MosqueraMM, MamouMB, et al.Long-term transmission of measles virus in Central and continental Western Europe. Virus Genes. 2015. [Epub ahead of print]. http://dx.doi.org/10.1007/s11262-0151173-1 PMID:25663095

11. Pegorie M, Shankar K, Welfare WS, Wilson RW, Khiroya C Munslow G, et al. Measles outbreak in Greater Manchester, England, October 2012 to September 2013: epidemiology and control. Euro Surveill. 2014;19(49):20982. http://dx.doi. org/10.2807/1560-7917.ES2014.19.49.20982 PMID:25523970 